\title{
Neuroprotective effects of ginsenosides on neural progenitor cells against oxidative injury
}

\author{
JUN YE $^{1 *}$, JIAN-PING YAO ${ }^{2 *}, \mathrm{XU} \mathrm{WANG}^{3}$, MINYING ZHENG $^{4}, \mathrm{PENG} \mathrm{LI}^{3}$, \\ CHENGWEI HE ${ }^{3}$, JIAN-BO WAN ${ }^{3}$, XIAOLI YAO ${ }^{4}$ and HUANXING SU ${ }^{3}$
}

\begin{abstract}
${ }^{1}$ Department of Dermatology, Sir Run Run Shaw Hospital, Zhejiang University School of Medicine, Hangzhou, Zhejiang 310016; ${ }^{2}$ Department of Cardiac Surgery II, The First Affiliated Hospital Sun Yat-Sen University, Guangzhou, Guangdong 510080; ${ }^{3}$ State Key Laboratory of Quality Research in Chinese Medicine,

Institute of Chinese Medical Sciences, University of Macau, Macau SAR 999078; ${ }^{4}$ Department of Neurology, National Key Clinical Department and Key Discipline of Neurology, The First Affiliated Hospital, Sun Yat-Sen University, Guangzhou, Guangdong 510080, P.R. China
\end{abstract}

Received March 18, 2015; Accepted January 20, 2016

DOI: $10.3892 / \mathrm{mmr} .2016 .4914$

\begin{abstract}
Ginsenosides exhibit various neuroprotective effects against oxidative stress. However, which ginsenoside provides optimal effects for the treatment of neurological disorders as a potent antioxidant remains to be elucidated. Therefore, the present study investigated and compared the neuroprotective effects of the Rb1, Rd, Rg1 and Re ginsenosides on neural progenitor cells (NPCs) following tert-Butylhydroperoxide ( $t$-BHP)-induced oxidative injury. Primary rat embryonic cortical NPCs were prepared from E14.5 embryos of Sprague-Dawley rats. The oxidative injury model was established with $t$-BHP. A lactate dehydrogenase assay and terminal deoxynucleotidyl transferase dUTP nick-end labeling staining were used to measure the viability of the NPCs pre-treated
\end{abstract}

Correspondence to: Professor Huanxing Su, State Key Laboratory of Quality Research in Chinese Medicine, Institute of Chinese Medical Sciences, University of Macau, N22 University Road, Macau SAR 999078, P.R. China

E-mail: huanxingsu@umac.mo

*Contributed equally

Abbreviations: Keap1, kelch-like ECH-associated protein1; ARE, antioxidant response element; CAT, catalase; HO-1, heme oxygenase-1; LDH, lactate dehydrogenase; Nrf2, nuclear factor (erythroid-derived 2)-like 2; NQO1, NAD(P) $\mathrm{H}$ dehydrogenase (quinone 1); SOD2, superoxide dismutase2; PLL, Poly-l-lysine; RT-qPCR, reverse transcription-quantitative polymerase chain reaction; $t$-BHP, tert-Butylhydroperoxide; CNS, central nervous system; NPCs, neural progenitor cells; BrdU, 5-Bromo-2-deoxyuridine; TUNEL, terminal deoxynucleotidy transferase dUTP nick-end labeling; GFAP, glial fibrillary acidic protein

Key words: ginsenosides, neural progenitor cells, oxidative injury with ginsenosides under oxidative stress. Reverse transcription-quantitative polymerase chain reaction analysis was used to determine the activation of intracellular signaling pathways triggered by the pretreatment of ginsenosides. Among the four ginsenosides, only $\mathrm{Rb} 1$ attenuated $t$-BHP toxicity in the NPCs, and the nuclear factor (erythroizd-derived 2)-like 2/heme oxygenase-1 pathway was found to be key in the intracellular defense against oxidative stress. The present study demonstrated the anti-oxidative effects of ginsenoside $\mathrm{Rb} 1$ on NPCs, and suggested that Rb1 may offer potential as a potent antioxidant for the treatment of neurological disorders.

\section{Introduction}

Neurological disorders affect $\sim 30,000,000$ individuals in China, leading to disability and contributing to mortality rates (1). These disorders are characterized by pathological changes in disease-specific areas of the brain, and the degeneration of distinct neural subsets (2). It has been well reported that neurological disorders are linked to elevated levels of oxidative stress, which is involved in modulating the biochemical changes resulting in neurological disorders (3). The supplementation of natural antioxidants is regarded as a prophylactic strategy against diseases caused by oxidative stress (4).

Ginseng, the root of Panax species $(5,6)$, is one of the most frequently used herbs in China due to its potential as a general tonic or chemopreventive agent $(7,8)$. The antioxidant action of ginseng is an area of interest in scientific investigations, which provides information for dietary supplementation and the pharmacological usage of ginseng products (9). Ginseng has been shown to have several beneficial effects in a wide range of pathological conditions, including cardiovascular disease, cancer, immunodeficiency and hepatotoxicity in vivo and in vitro. Of note, ginsenosides are the most biologically active substances found in ginseng (10). There are $>30$ different types of ginsenosides, which have been isolated from ginseng and classified into three major types: Panaxadiol, including Rb1, 
Rb2, Rg3, Rd, Rc, Rg3, Rh2 and Rs1); panaxatriol, including $\mathrm{Rg} 1, \operatorname{Rg} 2, \mathrm{Re}, \mathrm{Rf}$ and Rh1; and oleanolic acid type ginsenosides, including Ro (9). Among these, the most commonly investigated ginsenosides are Rb1, Rd, Rg1 and Re, as these four compounds are relatively more abundant in ginseng and have a wide range of actions in the central nervous system (CNS), including promoting neural survival, extending neurite growth and rescuing neurons from pathological conditions (11).

Several studies have provided evidence that ginsenoside $\mathrm{Rb} 1$ possesses potent neuroprotective effects on cortical neurons and dopaminergic neurons against glutamate toxicity, protects against cerebral ischemia by promoting neurogenesis, prevents $\mathrm{MPP}^{+}$-induced apoptosis in PC12 cells, improves spatial learning, and increases levels of hippocampal synaptophysin in mice (12-16). In the CNS, Rd has been shown to be effective in decreasing the formation of reactive oxygen species (ROS) in cultured astrocytes, protecting PC12 cells from hydrogen peroxide-induced oxidative damage, mitigating neuroinflammation and nitric oxide overproduction, and attenuating neuronal oxidative damage induced by oxygen-glucose deprivation (17). $\operatorname{Rg} 1$ has been shown to possess neurotrophic and neuroprotective effects on dopaminergic cells against glutamate injury and $\mathrm{MPP}^{+}$toxicity, inhibit the mitochondrial apoptotic pathway and increase the survival of primary cultured nigral neurons against rotenone toxicity (18). It has also been demonstrated that Rg1 exerts neuroprotective effects through ameliorating amyloid pathology, modulating the production of APP and activating the protein kinase A/ cAMP response element binding protein signaling pathways (19). Re has been reported to protect mouse nigral neurons from mitochondrial permeability transition pore-induced apoptosis in a Parkinson's disease model, and this effect was considered to be attributable to upregulation in the protein expression of $\mathrm{B}$ cell lymphoma (Bcl)-2, downregulation in the expression levels of $\mathrm{Bcl} 2$-associated $\mathrm{X}$ protein and inducible nitric oxide synthase, and subsequent inhibition of the activation of caspase-3 (20). These previous reports suggest that the $\mathrm{Rb}, \mathrm{Rd}, \mathrm{Rg} 1$ and $\mathrm{Re}$ ginsenosides offer therapeutic potential in the treatment of neurological disorders.

In the present study, the anti-oxidative effects of four ginsenosides (Rb1, Rd, Rg1 and Re) on NPCs were investigated and compared. NPCs can be utilized for functional tissue engineering as a potential treatment for neurologic diseases (21). They are defined by their ability to self-renew through mitotic cell division and differentiate into neurons, astrocytes and oligodendrocytes $(22,23)$. The results of the present study may provide evidence on the optimal ginsenoside for use as a potent antioxidant in the treatment of neurological disorders.

\section{Materials and methods}

Chemicals and reagents. Ginsenosides Rb1, $\mathrm{Rg} 1, \mathrm{Rd}$ and $\mathrm{Re}$ were provided in powder form ( $>98 \%$ purity) by Chengdu Must Bio-technology Co., Ltd. (Chengdu, China). The powder was dissolved in saline. Dulbecco's modified Eagle's medium (DMEM) nutrient mix F12, goat serum, fetal bovine serum (FBS), $0.05 \%$ (w/v) trypsin/EDTA, phosphate-buffered saline (PBS) powder and N2 and B27 supplements were supplied by Gibco (Thermo Fisher Scientific, Inc., Waltham, MA, USA).
Poly-1-lysine (PLL), laminin, 4',6-diamidino-2-phenylindole (DAPI), bovineserumalbumin(BSA),5-Bromo-2-deoxyuridine (BrdU), tert-Butylhydroperoxide ( $t$-BHP), paraformaldehyde, mouse anti-BrdU (B8434), rabbit anti-glial fibrillary acidic protein (GFAP; SAB4501162) and mouse anti- $\beta$-tubulin III (Tuj-1; T8578) were purchased from Sigma-Aldrich (St. Louis, MO, USA). A Lactate Dehydrogenase (LDH) Cytotoxicity Assay kit (cat. no. 11644793001) and In Situ Cell Death Detection kit (cat. no. 11684817910) were obtained from Roche (Basel, Switzerland). The goat anti-mouse 488 antibody, goat anti-rabbit 568 antibody, Click-iT EdU Alexa Fluor ${ }^{\circledR} 594$ Imaging kit (cat. no. C10339) and the Qubit ${ }^{\circledR}$ RNA BR Assay kit (cat. no. Q10210) were purchased from Invitrogen (Thermo Fisher Scientific, Inc.). The RNeasy ${ }^{\circledR}$ Mini kit (cat. no. 74134) was purchased from Qiagen (Hilden, Germany), and the primers, PrimeScript ${ }^{\mathrm{TM}}$ RT Master Mix (Perfect Real Time) kit (cat. no. RR036A) and SYBR ${ }^{\circledR}$ Premix Ex Taq ${ }^{\mathrm{TM}}$ II (Tli RNase H Plus; cat. no. RR820A) were supplied by Takara Biotechnology, Co., Ltd. (Dalian, China). The MicroAmp ${ }^{\circledR}$ Optical 96-well reaction plates with barcode were obtained from Applied Biosystems (Thermo Fisher Scientific, Inc.). Mouse anti-Nestin (MAB353) was purchased from EMD Milipore (Billerica, MA, USA); epidermal growth factor (EGF) and basic fibroblast factor (bFGF) were purchased from Peprotech (Rocky Hill, NJ, USA); and mouse anti-receptor interacting protein (Rip) was provided by Dr Xiaoming $\mathrm{Xu}$ of the University of Louisville (Louisville, USA). All other chemicals and reagents were of analytical grade.

Primary culture of cortical NPCs. A total of eight pregnant female Sprague-Dawley (SD) rats (weight, 300-350 g; age, 3-4 months) were obtained from the Animal Unit at the University of Macau (Macau, China). The rats were maintained in a temperature-controlled room under a 12-h light/dark cycle, with ad libitum access to food and water. The present study was approved by the Committee on the Care and Use of Laboratory Animals at the University of Macau (Macau, China). The primary rat embryonic cortical NPCs were prepared from E14.5 embryos derived from the SD rats using a modified protocol $(24,25)$. Briefly, the cortex was separated from the surrounding tissue following removal of the meninges. The cortex was transferred into a $15 \mathrm{ml}$ centrifuge tube containing culture medium $(10 \mu \mathrm{l} / \mathrm{ml} \mathrm{N} 2,20 \mu \mathrm{l} / \mathrm{ml} \mathrm{B} 27$, $20 \mathrm{ng} / \mathrm{ml} \mathrm{EGF}$ and $20 \mathrm{ng} / \mathrm{ml} \mathrm{bFGF} \mathrm{in} \mathrm{DMEM/F12)} \mathrm{and} \mathrm{disso-}$ ciated into a single-cell suspension $\left(5 \times 10^{6}\right.$ cells $\left./ \mathrm{ml}\right)$ by gentle mechanical trituration through a fire-polished Pasteur pipette. The dissociated cells were filtered through a cell strainer and then cultured in a T25 flask in suspension. The cells were incubated in a humidified incubator at $37^{\circ} \mathrm{C}$ in $5 \% \mathrm{CO}_{2}$. Half of the culture medium was replaced every 2-3 days. After 5-6 days, the cells had grown in neurospheres with the diameter of $\sim 150 \mu \mathrm{m}$. The cells in the neurospheres were passaged at the ratio of 1:6. These sub-cultured cells were designated as 'first passage' (P1) cells. The third passage (P3) cells were used for all subsequent experiments.

Establishment of the oxidative injury model. $t$-BHP is commonly used as a model substance for evaluating the mechanisms of cellular alterations resulting from oxidative stress in cells and tissues (26). In the present study, the P3 NPCs 
were dissociated into single cells, and then seeded into 96-well plates coated with PLL $(25 \mu \mathrm{g} / \mathrm{ml})$ and laminin $(13.3 \mu \mathrm{g} / \mathrm{ml})$ at a density of $1 \times 10^{4}$ cells per well. The cultures were grown at $37^{\circ} \mathrm{C}$ humidified $\mathrm{CO}_{2}$ incubator for $36 \mathrm{~h}$ and then treated with $50,100,200$ and $300 \mu \mathrm{M} t$-BHP for $2.5 \mathrm{~h}$ at $37^{\circ} \mathrm{C}$. The cytotoxicity of $t$-BHP in the whole cells culture was determined using an LDH cytotoxicity assay. A toxicity rate of $\sim 35-45 \%$ induced by $t$-BHP at specific concentrations was considered to be an optimal $t$-BHP-induced oxidative injury model.

Drug treatment. To determine the neuroprotective effects of the four ginsenosides, the cultured NPCs were pre-treated

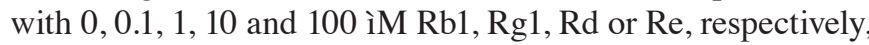
for $24 \mathrm{~h}$ at $37^{\circ} \mathrm{C}$, followed by drug washout with $0.01 \mathrm{M}$ PBS. The cells were then treated with $300 \mathrm{iM} t$-BHP for another $2.5 \mathrm{~h}$. The cell viability was measured using the LDH assay and further confirmed using a terminal deoxynucleotidyl transferase dUTP nick-end labeling (TUNEL) assay.

TUNEL assay. The free 3-OH DNA ends were detected in situ using an In Situ Cell Death Detection kit, according to the manufacturer's protocol. Briefly, after washing the cells three times with ice-cold PBS, the cells were fixed by incubation with fixation solution (Sigma-Aldrich) for $1 \mathrm{~h}$, followed by incubation with permeabilization solution (Sigma-Aldrich) for $2 \mathrm{~min}$ on ice. The fixed cell samples were incubated in the TUNEL reaction medium for $1 \mathrm{~h}$ at $37^{\circ} \mathrm{C}$ in the dark. Following completion of the reaction, the cells were washed using PBS, transferred into $2 \mu \mathrm{g} / \mathrm{ml}$ DAPI solution, and mounted on slides. The number of apoptotic nuclei and the total number of nuclei were determined under a fluorescence microscope (Axio Imager A2; Carl Zeiss AG, Oberkochen, Germany).

Lactate dehydrogenase $(\mathrm{LDH})$ release assay. Cell death in the NPCs was quantified by measuring the release of LDH into the medium. As the enzyme is released from cells with damaged membranes, the efflux of LDH is closely associated with the extent of damage or destruction of the NPCs (27). To confirm cortical NPC injury, the activity of LDH in the medium following oxidative injury was determined using the Cytotoxicity Detection kit, according to the manufacturer's protocol. Briefly, the treated cells were lysed for $45 \mathrm{~min}$ at $37^{\circ} \mathrm{C}$ in PBS supplemented with $1 \mathrm{X}$ Triton X-100 (0.1\%; Invitrogen; Thermo Fisher Scientific, Inc.), followed by centrifugation at $1,000 \mathrm{x} \mathrm{g}$ for $10 \mathrm{~min}$ at $37^{\circ} \mathrm{C}$. The sample supernatants were transferred to a 96-well enzymatic assay plate and reacted with the substrate mix from the Cytotoxicity Detection kit in the dark for $30 \mathrm{~min}$ at room temperature. The absorbance of the samples was measured at $490 \mathrm{~nm}$, according to the filter of the SpectraMax ${ }^{\mathrm{R}}$ M5 Multi-Mode microplate reader (Molecular Devices LLC, Sunnyvale, CA, USA). Each experiment was repeated three times independently.

Immunocytochemical analysis. The P3 NPCs in a single cell suspension were cultured on cover slips, which were coated with PLL/laminin (1:1 ratio), at a density of $1 \times 10^{4}$ cells $/ \mathrm{cm}^{2}$ in a 24-well plate. For differentiation experiments, growth factors were removed from the culture medium and 1\% FBS was added. The cultures were allowed to differentiate for up to 5 days.
The cells on the cover slips were then fixed with freshly prepared $4 \%$ paraformaldehyde solution in PBS at room temperature for $20 \mathrm{~min}$. Following several washes with $0.01 \mathrm{M} \mathrm{PBS}$, the cells were processed for immunocytochemistry. The following primary antibodies were used to stain the cells: Monoclonal anti-Nestin antibody (1:500) for NPCs; monoclonal anti-Tuj-1 antibody (1:500) for neurons; polyclonal anti-GFAP antibody $(1: 1,000)$ for astrocytes; and monoclonal anti-Rip antibody (1:50) for oliogodendrocytes. The cultures were incubated with the primary antibodies in PBS with 1\% BSA, 10\% normal goat serum and $0.3 \%$ Triton $\mathrm{X}-100$ overnight at $4^{\circ} \mathrm{C}$. The samples were then washed twice with PBS and incubated in secondary antibody conjugated to fluorescent Alexa 568 or 488 (1:500; Thermo Fisher Scientific, Inc.) for $45 \mathrm{~min}$ at room temperature. To visualize the nuclei, the cells were mounted in anti-fade solution containing DAPI for $10 \mathrm{~min}$. The fluorescence images were captured using a fluorescence microscope.

Reverse transcription-quantitative polymerase chain reaction (RT-qPCR) analysis. RT-qPCR was used to evaluate the mRNA expression levels of the antioxidant gene in response to oxidative injury. The cells were pretreated with $10 \mu \mathrm{M} \mathrm{Rb1}$ for $24 \mathrm{~h}$, after which total RNA was extracted using the RNeasy ${ }^{\circledR}$ Mini kit. The RNA concentrations were determined using a NanoDrop 2000 (Thermo Fisher Scientific, Inc.) with a Qubit ${ }^{\circledR}$ RNA BR Assay kit. Total RNA was reverse transcribed into cDNA using the PrimeScript ${ }^{\mathrm{TM}}$ RT Master Mix kit, according to the manufacturer's protocol. Amplifications were performed in duplicate in $20 \mu \mathrm{l}$ reaction volumes containing $1 \mathrm{X} \mathrm{SYBR}{ }^{\circledR}$ Premix Ex Taq ${ }^{\mathrm{TM}}$ II (Tli RNase H Plus), $0.2 \mu \mathrm{M}$ of each primer and $2 \mu 1$ target DNA, to quantitatively detect the gene expression levels of nuclear factor (erythroid-derived 2)-like 2 (Nrf2), heme oxygenase-1 (HO-1), superoxide dismutase 2 (SOD2), NAD(P)H: quinone oxidoreductase 1 (NQO1) and catalase (CAT). The relative expression level of each target gene was normalized to the housekeeping gene, $\beta$-actin. All primer sequences used are listed in Table I. Subsequently, qPCR was performed using the 7500 Real-Time PCR system (Applied Biosystems; Thermo Fisher Scientific, Inc.). The reaction conditions were as follows: Initial denaturation at $95^{\circ} \mathrm{C}$ for $30 \mathrm{sec}$, followed by 40 cycles of $95^{\circ} \mathrm{C}$ for $5 \mathrm{sec}$ and $60^{\circ} \mathrm{C}$ for $34 \mathrm{sec}$, followed by melting curve analysis. Each sample was assessed in triplicate and the $2^{-\Delta \Delta \mathrm{Cq}}$ method was used to analyze the relative transcription data (28).

Statistical analysis. All data are expressed as the mean \pm standard deviation. Statistical analyses were performed using SPSS software, version 17.0 (SPSS, Inc., Chicago, IL, USA). The two-tailed Student's t-test was used to make comparisons between two groups and one-way analysis of variance followed by Tukey's post-hoc test was used to analyze differences among multiple groups. $\mathrm{P}<0.05$ was considered to indicate a statistically significant difference.

\section{Results}

Characterization of NPCs. In the presence of the EGF and bFGF mitogens, the majority of cells showed bipolar or multipolar morphology with small cell bodies, and were immunoreactive 
Table I. Primer sequences for reverse transcription-quantitative polymerase chain reaction analysis.

\begin{tabular}{llll}
\hline Target gene & Direction & \multicolumn{1}{c}{ Sequence } & Association no. \\
\hline$\beta$-actin & Forward & 5'-GTCGTACCACTGGCATTCTG-3' & NM_031144 \\
& Reverse & 5'-CTCTCAGCTGTGGTGGTGAA-3' & \\
NRF-2 & Forward & 5'-GCAACTCCAGAAGGAACAGG-3' & NM-031789.1 \\
& Reverse & 5'-CAGTGAGGGGATCGATGAGT-3' & \\
HO-1 & Forward & 5'-TGCTCGCATGAACACTCTG-3' & NM_012580.2 \\
& Reverse & 5'-TCCTCTGTCAGCAGTGCCT & \\
SOD2 & Forward & 5'-GGCCAAGGGAGATGTTACAA-3' & NM_001274771 \\
NQO1 & Reverse & 5'-GCTTGATAGCCTCCAGCAAC-3' & \\
& Forward & 5'-GCCCGGATATTGTAGCTgAA-3' & NM_017000.3 \\
CAT & Reverse & 5'-GTGGTGATGGAAAGCAAGGT-3' & \\
& Forward & 5'-TTATGGCCTCCGAGATCTTTTC-3' & NM_012520 \\
\hline
\end{tabular}

NRF-2, nuclear factor (erythroid-derived 2)-like 2; HO-1, heme oxygenase-1; SOD2, superoxide dismutase 2; NQO1, NAD(P)H: quinone oxidoreductase 1; CAT, catalase.

A

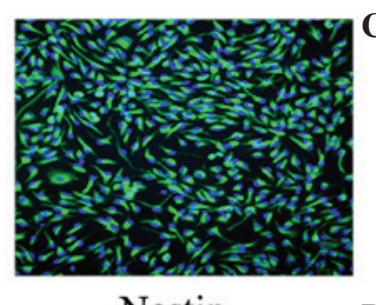

B

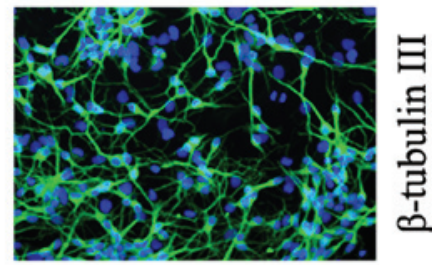

C

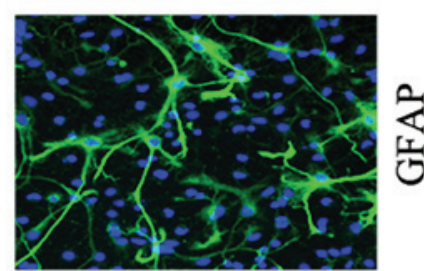

D

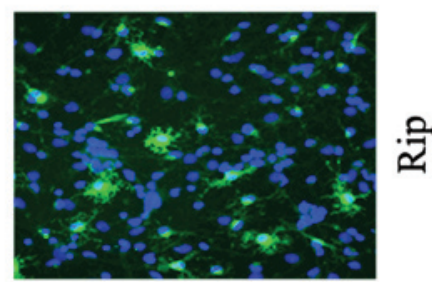

Figure 1. Characterization on NPCs. (A) Image shows P3 NPCs cultured in basic fibroblast factor-and epidermal growth factor-supplemented culture medium. Cells were either bipolar or multipolar with immunoreactivity for Nestin. When cultured in differentiating medium, the NPCs differentiated into (B) $\beta$-tubulin III-positive neurons, (C) GFAP-positive astrocytes and (D) Rip-positive oligodendrocytes. Scale bar=150 $\mu \mathrm{m}$ in A; $50 \mu \mathrm{m}$ in B-D. NPCs, neural progenitor cells; GFAP, glial fibrillary acidic protein; Rip, receptor interacting protein.

for Nestin, an effective marker for NPCs (Fig. 1A), confirming that the cells remained in an immature stage. Following replacement of the mitogens with $1 \%$ FBS, the NPCs began to differentiate. At day 5 of culture in the differentiating medium, the NPCs had successfully differentiated into Tuj-1-positive neurons (Fig. 1B), GFAP-positive astrocytes (Fig. 1C) and Rip-positive oligodendrocytes (Fig. 1D).

Neuroprotective effects of the four ginsenosides on $t$-BHP-induced cytotoxicity in NPCs. The present study used
$t$-BHP to establish a model of oxidative injury. As shown in Fig. 2A, $t$-BHP treatment induced cell toxicity in a concentration-dependent manner. The NPCs treated with 50, 100, 200 and $300 \mu \mathrm{M}$ for $2.5 \mathrm{~h}$ exhibited a cytotoxicity rate of $5.43 \pm 1.40$, $9.07 \pm 2.20,13.13 \pm 1.80$ and $37.67 \pm 2.52 \%$, respectively. As a toxicity rate of $35-45 \%$ induced by $t$-BHP was considered to be an optimal oxidative stress model, the oxidative injury induced by $300 \mu \mathrm{M} t$-BHP for $2.5 \mathrm{~h}$ was selected for the subsequent experiments to investigating the anti-oxidative effect of the four ginsenosides. 
A

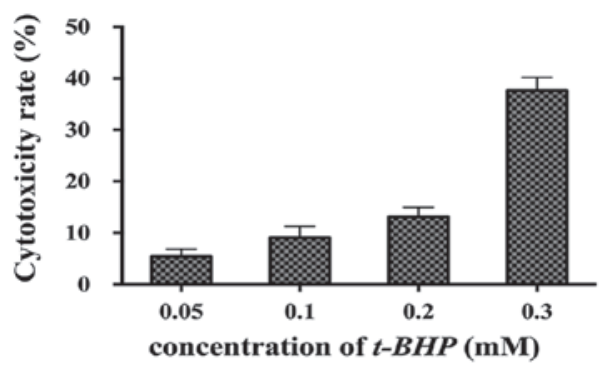

B

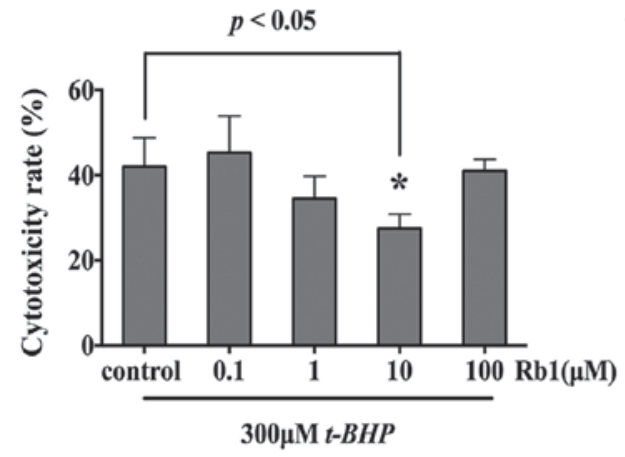

D

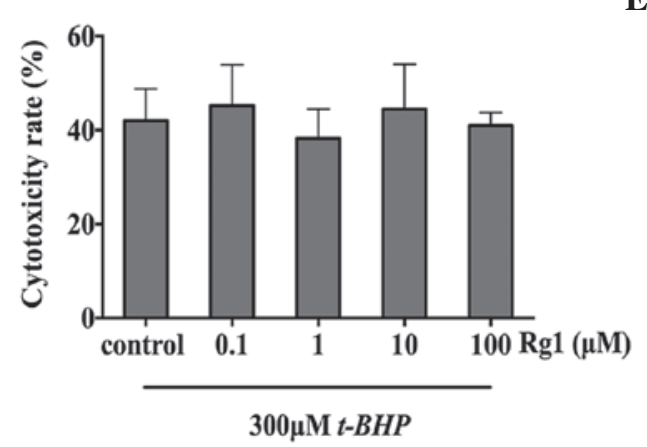

C

$\mathbf{E}$
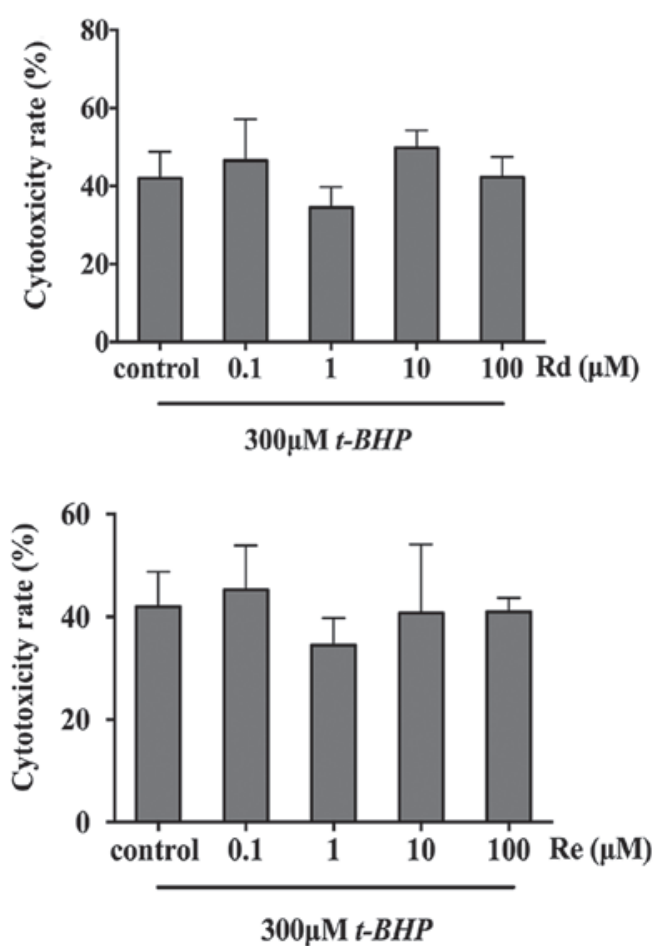

Figure 2. Evaluation of the effects of the Rb1, Rd, Rg1 and Re ginsenosides on $t$-BHP-induced cytotoxicity in NPCs using LDH assay. (A) Establishment of $t$-BHP-induced oxidative injury model. (B) LDH assays revealed that pretreatment with $10 \mu \mathrm{M}$ Rb1 for $24 \mathrm{~h}$ significantly reduced oxidative stress on the NPCs. Results are expressed as the mean \pm standard deviation $(n=4)$, $P<0.05$, vs. control group. LDH assays revealed that pretreatment with $(\mathrm{C}) \mathrm{Rd}$, (D) Rg1 and (E) Re at different concentrations showed no anti-oxidative effects on the NPCs. NPCs, neural progenitor cells; $t$-BHP; tert-Butylhydroperoxide; LDH, lactate dehydrogenase.

The NPCs were pretreated with different concentrations of Rb1, Rd, Rg1, Re $(0.1,1,10$ and $100 \mu \mathrm{M})$ for $24 \mathrm{~h}$, followed by treatment with $300 \mu \mathrm{M} t$-BHP for $2.5 \mathrm{~h}$, respectively. Cell viability was measured using an LDH assay and TUNEL staining. The results of the LDH assay suggested that only $10 \mu \mathrm{M}$ $\mathrm{Rb} 1$ showed a protective effect against oxidative stress, with a cytotoxicity rate of $27.5 \pm 2.87 \%$ in the $10 \mu \mathrm{M}$ Rb1-pretreated group, compared with $42 \pm 5.87 \%$ in the control group $(\mathrm{P}=0.0208$; Fig. 2B). Rd, Rg1 and Re had no neurooprotective effects against oxidative injury (Fig. 2C-E). The neuroprotective effects of Rb1 were confirmed by the TUNEL assay, with an apoptotic index of $12.5 \pm 2.20 \%$ in the $10 \mu \mathrm{M}$ Rb1-pretreated group, compared with $23 \pm 3.02 \%$ in the control group ( $\mathrm{P}=0.0003$; Fig. $3 \mathrm{~A}-\mathrm{C})$. The TUNEL staining demonstrated that the remaining three ginsenosides, Rd, Rg1 and Re, exhibited no neuroprotective effects on the NPCs against oxidative injury (Fig. 3D-F).

Rb1 pretreatment activates anti-oxidative genes in cultured NPCs. The present study subsequently investigated the potential mechanism underlying the anti-oxidative effect induced by Rb1. Firstly, the changes in the mRNA expression levels of Nrf2 were measured using RT-qPCR analysis. Nrf2 belongs to the basic-leucine zipper family and coordinately upregulates the constitutive and inducible transcription of a wide array of genes involved in drug metabolism, detoxification and antioxidant defenses (29). The mRNA expression level of Nrf2 was increased 2-fold following pretreatment of the cultured NPCs with $10 \mu \mathrm{M}$ Rb1 for $24 \mathrm{~h}$. However, pretreatment of the NPCs with the other three ginsenosides did not elevate the expression of Nrf2 (Fig. 4A). The mRNA expression levels of the Nrf2-responsive genes, HO-1, SOD2, NQO1 and CAT were then examined. A 1.5-fold increase in the expression of HO-1 was observed in the $10 \mu \mathrm{M}$ Rb1-pretreated cells, whereas the other three ginsenosides had no effects on the activation of the downstream HO-1, SOD2, NQO1 or CAT genes (Fig. 4B).

\section{Discussion}

Ginseng is reported to have a wide range of therapeutic and pharmacological applications, and has been widely used to 

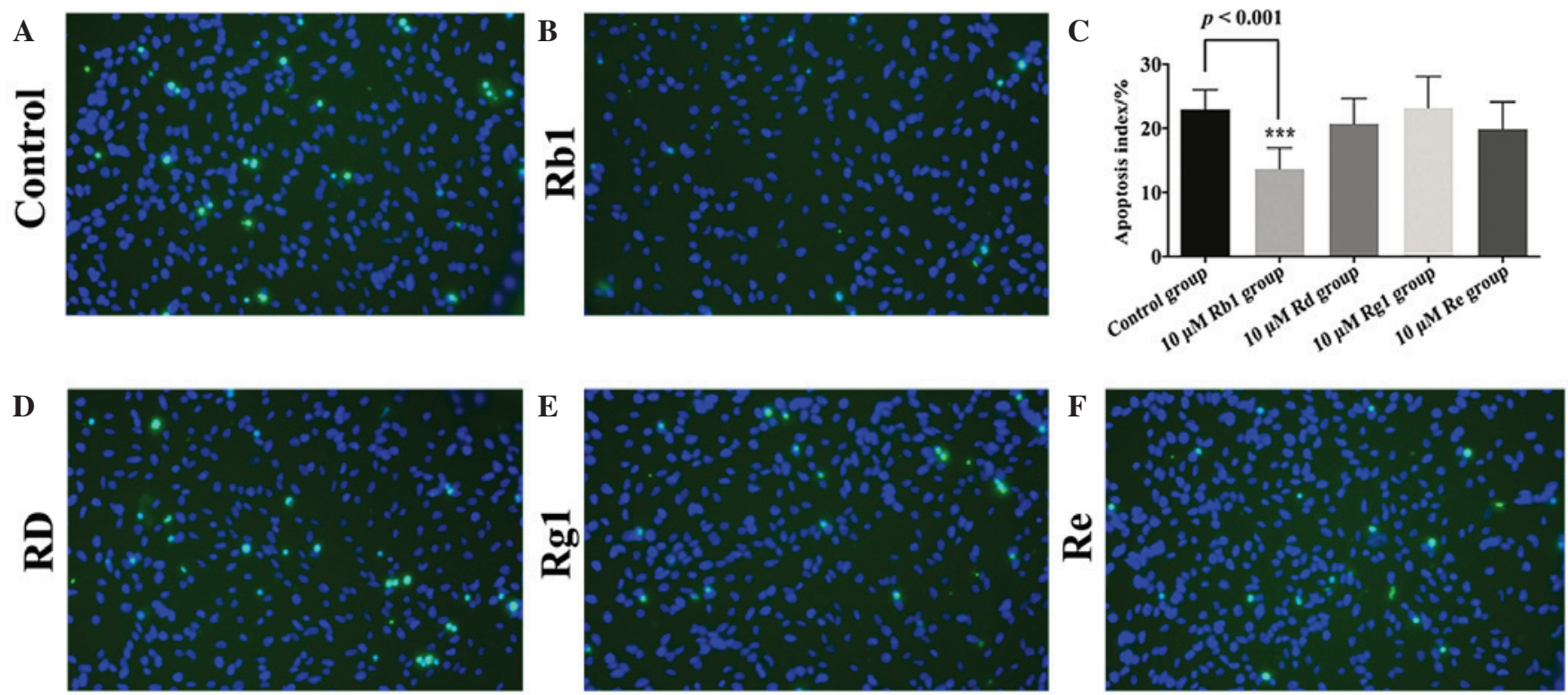

Figure 3. Pretreatment with $10 \mu \mathrm{M}$ Rb1 significantly reduces the apoptosis of NPCs induced by $t$-BHP-cytotoxicity. Representative photomicrographs of TUNEL staining in the (A) control group, (B) $10 \mu \mathrm{M}$ Rb1-treated group, (C) $10 \mu \mathrm{M}$ Rd-treated group, (D) $10 \mu \mathrm{M} \mathrm{Rg1-treated} \mathrm{group} \mathrm{and} \mathrm{(E)} 10 \mu \mathrm{M} \mathrm{Re-treated}$ group. (F) Quantitative analyses of the TUNEL staining confirmed that the percentage of TUNEL-positive cells was significantly reduced in the $10 \mu \mathrm{M}$ Rbl-treated group, compared with the control. Cell apoptosis index values are presented as the mean \pm standard deviation from six independent experiments. ${ }^{* * * *} \mathrm{P}<0.001$. Scale bar=200 $\mu \mathrm{m}$. NPCs, neural progenitor cells; $t$-BHP; tert-Butylhydroperoxide; TUNEL, terminal deoxynucleotidyl transferase dUTP nick-end labeling.
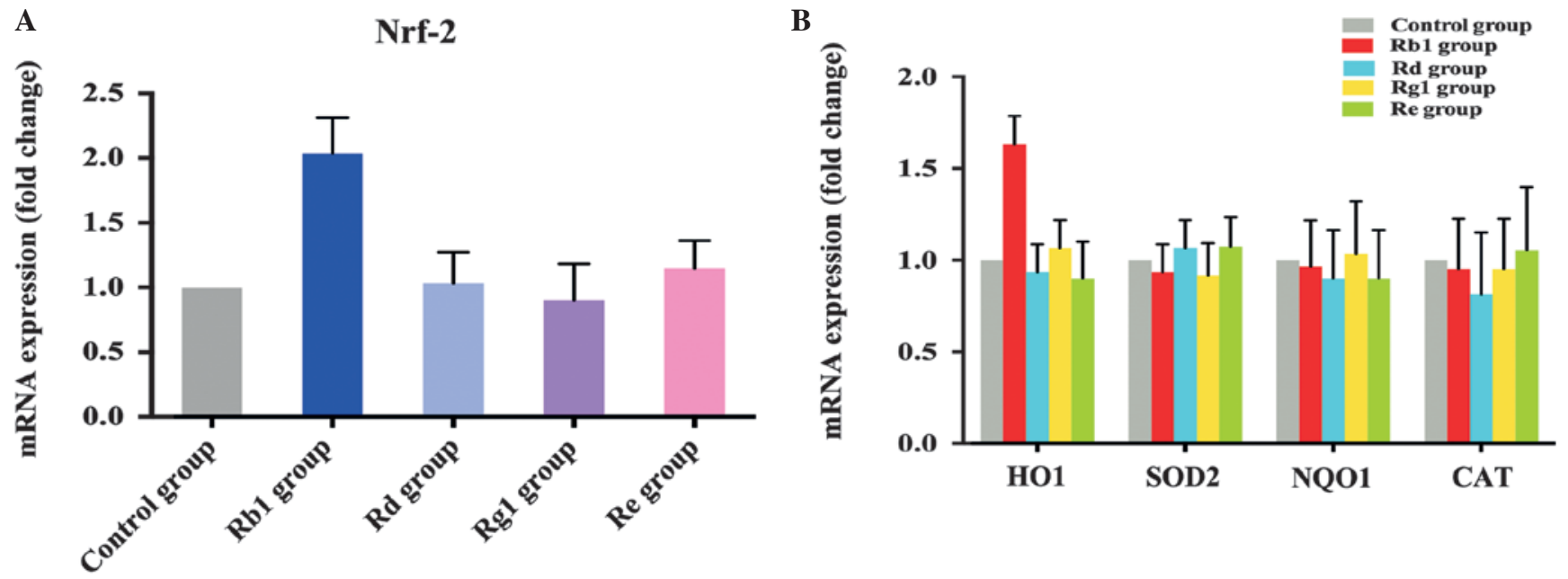

Figure 4. mRNA expression levels of Nrf2, HO-1, SOD2, NQO1 and CAT in NPCs following pretreatment with Rb1, Rd, Rg1 and Re. (A) RT-qPCR analysis demonstrated that the expression of Nrf2 was increased 2-fold in NPCs following pre-treatment with $10 \mu \mathrm{M} \mathrm{Rb1}$ for $24 \mathrm{~h}$, compared with the control, whereas no changes in the expression of Nrf2 were observed following pre-treatment with $10 \mu \mathrm{M} \mathrm{Rd}, 10 \mu \mathrm{M} \mathrm{Rg} 1$ or $10 \mu \mathrm{M} \mathrm{Re}$. (B) RT-qPCR measurement of levels of HO-1, SOD2, NQO1 and CAT in NPCs following pre-treatment with $10 \mu \mathrm{M} \mathrm{Rb1,} 10 \mu \mathrm{M} \mathrm{Rd}, 10 \mu \mathrm{M} \mathrm{Rg} 1$ and $10 \mu \mathrm{M}$ Re for $24 \mathrm{~h}$, followed by incubation with $300 \mu \mathrm{M} t$-BHP for $2.5 \mathrm{~h}$. Rb1 treatment significantly increased the expression of HO-1, compared with the control group, but did not increase the expression levels of SOD2, NQO1 or CAT. Rd, Rg1 and Re had no effect on the expression levels of HO-1, SOD2, NQO1 or CAT in the NPCs. NPCs, neural progenitor cells; $t$-BHP; tert-Butylhydroperoxide; Nrf-2, nuclear factor (erythroid-derived 2)-like 2; HO-1, heme oxygenase-1; SOD2, superoxide dismutase 2; NQO1, $\mathrm{NAD}(\mathrm{P}) \mathrm{H}$ : quinone oxidoreductase 1; CAT, catalase; RT-qPCR, reverse transcription-quantitative polymerase chain reaction.

treat various diseases and improve health for thousands of years in Asia (7). Accumulating evidence has indicated that ginsenosides are the principle pharmacologically active ingredients of ginseng. An increasing number of studies are being performed to investigate purified ginsenoside alone to examine the mechanism of function of ginseng, rather than using whole ginseng root (30-35). Each ginsenoside is suggested to have distinct effects in pharmacology and distinct mechanisms due to their unique structures (36). At present, $\sim 40$ ginsenoside compounds have been identified, among which Rbl, Rd, Rg1 and $R e$ are the most commonly investigated ginsenosides due to their quantitative abundance in ginseng root (9). The present study investigated and compared the neuroprotective effects of four types of ginsenosides on NPCs against oxidative stress. The results showed that only Rb1 exhibited a protective effect on the NPCs, whereas the Rd, Rg1 and Re ginsenosides 


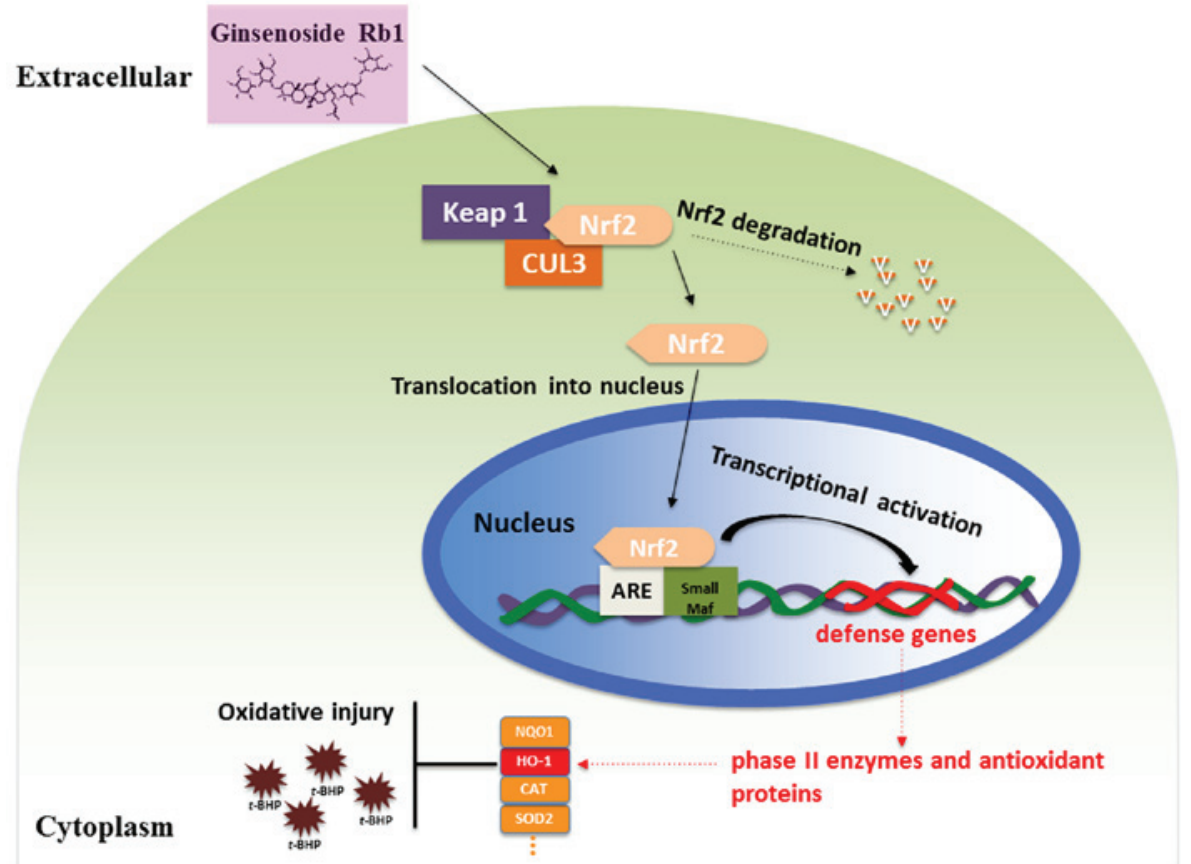

Figure 5. Suggested mechanisms underlying the cellular responses to oxidative injury and protective effects of Rb1 pre-treatment via the Nrf2/HO-1 signaling pathways. Keap1, kelch-like ECH-associated protein1; Maf, musculoaponeurotic fibrosarcoma; ARE, antioxidant response element; CAT, catalase; HO-1, heme oxygenase-1; Nrf2, nuclear factor (erythroid-derived 2)-like 2; NQO1, NAD(P)H dehydrogenase (quinone 1); SOD2, superoxide dismutase 2; CUL3, cullin 3 .

exhibited no protective effects towards NPCs under oxidative stress.

Oxidative stress is defined as the general principle of imbalance between the formation and detoxification of ROS. When not sufficiently scavenged, these small molecules may cause DNA damage, or mutations and lipid peroxidation, leading to membrane damage (37). Substantial evidence has indicated that oxidative stress is a major contributor to the pathophysiology of a variety of neurodegenerative disorders, including Alzheimer's disease, Parkinson's disease and acute CNS injuries, including spinal cord injury and traumatic brain injury $(2,38,39)$. Ginsenosides have been confirmed to exert protective effects, attributed to their antioxidant ability through increasing internal antioxidant enzymes and acting as a free-radical scavenger (40-42). It has been suggested that the administration of 100 or $200 \mathrm{mg} / \mathrm{kg} /$ day of ginsenosides through drinking water improves memory loss in senescence accelerated (SAMP8) mice, and increases serum antioxidant levels (43).

The association between the structure of ginsenoside and its anti-oxidative or pro-oxidative activity has been investigated in free radical-induced hemolysis of human erythrocytes $(44,45)$. The exact mechanisms underlying the differences in protective effects of ginsenosides on NPCs against oxidative stress remain to be elucidated. A number of studies have suggested that Rb1 has beneficial effects in the treatment of oxidative stress (46-51). The present study also demonstrated that pretreatment with Rb1 significantly protected NPCs against oxidative injury, and upregulated Nrf2 and its downstream antioxidant-responsive gene, HO-1. It is generally considered that the activation of the Nrf2 may further upregulate the transcription of multiple antioxidant response element (ARE)-controlled genes, and finally initiate the expression of a variety of antioxidant enzymes and phase II drug-metabolizing enzymes $(52,53)$. HO-1, which belongs to the heat shock protein family, is an inducible enzyme, which catalyzes the first and rate-limiting step in oxidative degradation (54). Evidence has indicated the critical role of HO-1 and its enzymatic by-products in anti-inflammation, anti-oxidation, and more diverse biological functions $(55,56)$.

The mechanism underlying the response of the Nrf2/HO-1 signaling pathway to oxidative stress on NPCs by pretreatment with Rb1 is shown in Fig. 5. Under homeostatic conditions, Nrf2 signaling is repressed by Kelch-like ECH-associated protein 1 (Keap1), which has been identified as a Cullin3-dependent substrate adaptor protein. Nrf2 is found to bind to Keap1 and be sequestered in the cytoplasm, where it is ubiquitinated and subsequently degraded (57). When treated with ginsenoside $\mathrm{Rb} 1, \mathrm{Nrf} 2$ is activated and triggered to translocate into the nucleus, where it elicits a series of anti-oxidative responses. A complex, which consists of Nrf2 protein, a group of small musculoaponeurotic fibrosarcoma proteins and a cis-acting enhancer, ARE, is then formed, which is essential for the anti-oxidative response to cell injury induced by $t$-BHP by activating the transcription of the downstream anti-oxidative gene, HO-1 $(58,59)$.

The present study provided an overview on the pharmacological activity of ginsenoside $\mathrm{Rb} 1, \mathrm{Rd}, \mathrm{Rg} 1$ and $\mathrm{Re}$, in terms of the neuroprotective effects on NPCs against oxidative injury. Only Rb1 was shown to have protective effects, by activating Nrf2/HO-1 pathway, in an experimental model of oxidative injury, whereas $\mathrm{Rd}, \operatorname{Rg} 1$ and $\mathrm{Re}$ had no protective effects on the NPCs against oxidative injury. Future investigations are warranted to further examine the mechanisms underlying the 
protective actions of ginsenoside Rb1 against oxidative injury, and to investigate the therapeutic potential of $\mathrm{Rbl}$ in animal models.

\section{Acknowledgements}

The present study was supported by a multi-year research grant from the University of Macau [grant nos. MYRG122 (Y1-L3)-ICMS12-SHXandMYRG110(Y1-L2)-ICMS13-SHX], a matching project grant (grant no. MRG003/SHX/2014/ICMS) and the Macao Science and Technology Development Fund (grant no. 018/2013/A1).

\section{References}

1. Pei JJ, Giron MS, Jia J and Wang HX: Dementia studies in Chinese populations. Neurosci Bull 30: 207-216, 2014.

2. Radi E, Formichi P, Battisti C and Federico A: Apoptosis and oxidative stress in neurodegenerative diseases. J Alzheimers Dis 42 (Suppl 3): S125-S152, 2014.

3. Ghaffari H, Venkataramana M, Jalali Ghassam B, Chandra Nayaka S, Nataraju A, Geetha NP and Prakash HS Rosmarinic acid mediated neuroprotective effects against HO-induced neuronal cell damage in N2A cells. Life Sci 113: 7-13, 2014.

4. Surh YJ: Cancer chemoprevention with dietary phytochemicals. Nat Rev Cancer 3: 768-780, 2003.

5. Diao Y, Lin XM, Liao CL, Tang CZ, Chen ZJ and Hu ZL: Authentication of Panax ginseng from its adulterants by PCR-RFLP and ARMS. Planta Med 75: 557-560, 2009.

6. Qin JH, Leung FC, Fung Y, Zhu D and Lin B: Rapid authentication of ginseng species using microchip electrophoresis with laser-induced fluorescence detection. Anal Bioanal Chem 381: 812-819, 2005

7. Lu JM, Yao Q and Chen C: Ginseng compounds: An update on their molecular mechanisms and medical applications. Curr Vasc Pharmacol 7: 293-302, 2009.

8. Rausch WD, Liu S, Gille G and Radad K: Neuroprotective effects of ginsenosides. Acta Neurobiol Exp (Wars) 66: 369-375, 2006.

9. Liu ZQ: Chemical insights into ginseng as a resource for natural antioxidants. Chem Rev 112: 3329-3355, 2012.

10. Ru W, Wang D, Xu Y, He X, Sun YE, Qian L, Zhou X and Qin Y: Chemical constituents and bioactivities of Panax ginseng (C. A. Mey.). Drug Discov Ther 9: 23-32, 2015.

11. Zhang YF, Fan XJ, Li X, Peng LL, Wang GH, Ke KF and Jiang ZL: Ginsenoside Rg1 protects neurons from hypoxic-ischemic injury possibly by inhibiting $\mathrm{Ca} 2+$ influx through NMDA receptors and L-type voltage-dependent Ca2+ channels. Eur J Pharmacol 586: 90-99, 2008.

12. Ni N, Liu Q, Ren H, Wu D, Luo C, Li P, Wan JB and Su H: Ginsenoside $\mathrm{Rb} 1$ protects rat neural progenitor cells against oxidative injury. Molecules 19: 3012-3024, 2014

13. Chen Z, Lu T, Yue X, Wei N, Jiang Y, Chen M, Ni G, Liu X and $\mathrm{Xu}$ G: Neuroprotective effect of ginsenoside Rb1 on glutamate-induced neurotoxicity: With emphasis on autophagy. Neurosci Lett 482: 264-268, 2010.

14. Luo T, Liu G, Ma H, Lu B, Xu H, Wang Y, Wu J, Ge P and Liang J: Inhibition of autophagy via activation of PI3K/Akt pathway contributes to the protection of ginsenoside Rb1 against neuronal death caused by ischemic insults. Int J Mol Sci 15: 15426-15442, 2014

15. Liu J, He J, Huang L, Dou L, Wu S and Yuan Q: Neuroprotective effects of ginsenoside Rb1 on hippocampal neuronal injury and neurite outgrowth. Neural Regen Res 9: 943-950, 2014.

16. Hashimoto R, Yu J, Koizumi H, Ouchi Y and Okabe T: Ginsenoside Rb1 Prevents MPP(+)-Induced Apoptosis in PC12 Cells by Stimulating Estrogen Receptors with Consequent Activation of ERK1/2, Akt and Inhibition of SAPK/JNK, p38 MAPK. Evid Based Complement Alternat Med 2012: 693717, 2012.

17. Ye R, Yang Q, Kong X, Han J, Zhang X, Zhang Y, Li P, Liu J, Shi M, Xiong L and Zhao G: Ginsenoside Rd attenuates early oxidative damage and sequential inflammatory response after transient focal ischemia in rats. Neurochem Int 58: 391-398, 2011
18. Leung KW, Yung KK, Mak NK, Chan YS, Fan TP and Wong RN: Neuroprotective effects of ginsenoside-Rg1 in primary nigral neurons against rotenone toxicity. Neuropharmacology 52: $827-835,2007$

19. Fang F, Chen X, Huang T, Lue LF, Luddy JS and Yan SS: Multi-faced neuroprotective effects of Ginsenoside Rg1 in an Alzheimer mouse model. Biochim Biophys Acta 1822: 286-292, 2012.

20. Xu BB, Liu CQ, Gao X, Zhang WQ, Wang SW and Cao YL: Possible mechanisms of the protection of ginsenoside $\mathrm{Re}$ against MPTP-induced apoptosis in substantia nigra neurons of Parkinson's disease mouse model. J Asian Nat Prod Res 7: 215-224, 2005

21. Leipzig ND, Wylie RG, Kim H and Shoichet MS: Differentiation of neural stem cells in three-dimensional growth factor-immobilized chitosan hydrogel scaffolds. Biomaterials 32: 57-64, 2011.

22. Gage FH: Mammalian neural stem cells. Science 287: 1433-1438, 2000.

23. Alvarez-Buylla A and Garcia-Verdugo JM: Neurogenesis in adult subventricular zone. J Neurosci 22: 629-634, 2002.

24. Reynolds BA and Weiss S: Generation of neurons and astrocytes from isolated cells of the adult mammalian central nervous system. Science 255: 1707-1710, 1992.

25. Su HX, Zhang W, Guo J, Guo A, Yuan Q and Wu W: Neural progenitor cells enhance the survival and axonal regeneration of injured motoneurons after transplantation into the avulsed ventral horn of adult rats. J Neurotrauma 26: 67-80, 2009.

26. Kučera O, Endlicher R, Roušar T, Lotková H, Garnol T, Drahota Z and Cervinková Z: The effect of tert-butyl hydroperoxide-induced oxidative stress on lean and steatotic rat hepatocytes in vitro. Oxid Med Cell Longev 2014: 752506, 2014.

27. Koh JY and Choi DW: Quantitative determination of glutamate mediated cortical neuronal injury in cell culture by lactate dehydrogenase efflux assay. J Neurosci Methods 20: 83-90, 1987.

28. Livak KJ and Schmittgen TD: Analysis of relative gene expression data using real-time quantitative PCR and the $2-\Delta \Delta \mathrm{Ct}$ method. Methods 25: 402-408, 2001.

29. Aueviriyavit S, Phummiratch D and Maniratanachote R: Mechanistic study on the biological effects of silver and gold nanoparticles in Caco-2 cells-induction of the $\mathrm{Nrf} 2 / \mathrm{HO}-1$ pathway by high concentrations of silver nanoparticles. Toxicol Lett 224: 73-83, 2014.

30. Buettner C, Yeh GY, Phillips RS, Mittleman MA and Kaptchuk TJ: Systematic review of the effects of ginseng on cardiovascular risk factors. Ann Pharmacother 40: 83-95, 2006.

31. Gillis CN: Panax ginseng pharmacology: A nitric oxide link? Biochem Pharmacol 54: 1-8, 1997.

32. Hofseth LJ and Wargovich MJ: Inflammation, cancer and targets of ginseng. J Nutr 137 (Suppl 1): 183S-185S, 2007.

33. Attele AS, Wu JA and Yuan CS: Ginseng pharmacology: Multiple constituents and multiple actions. Biochem Pharmacol 58: 1685-1693, 1999.

34. Zhou W, Chai H, Lin PH, Lumsden AB, Yao Q and Chen CJ: Molecular mechanisms and clinical applications of ginseng root for cardiovascular disease. Med Sci Monit 10: RA187-RA192, 2004.

35. Cheng Y, Shen LH and Zhang JT: Anti-amnestic and anti-aging effects of ginsenoside Rg1 and Rb1 and its mechanism of action. Acta Pharmacol Sin 26: 143-149, 2005.

36. Murthy HN, Georgiev MI, Kim YS, Jeong CS, Kim SJ, Park SY and Paek KY. Ginsenosides: Prospective for sustainable biotechnological production. Appl Microbiol Biotechnol 98: 6243-6254, 2014.

37. Alfadda AA and Sallam RM: Reactive oxygen species in health and disease. J Biomed Biotechnol 2012: 936486, 2012

38. Smith JA, Park S, Krause JS and Banik NL: Oxidative stress, DNA damage and the telomeric complex as therapeutic targets in acute neurodegeneration. Neurochem Int 62: 764-775, 2013.

39. Fernández-Gajardo R, Matamala JM, Carrasco R, Gutiérrez R, Melo R and Rodrigo R: Novel therapeutic strategies for traumatic brain injury: Acute antioxidant reinforcement. CNS Drugs 28: 229-248, 2014.

40. Xie JT, Shao ZH, Hoek TL, Chang WT, Li J, Mehendale S, Wang CZ, Hsu CW, Becker LB, Yin JJ and Yuan CS: Antioxidant effects of ginsenoside Re in cardiomyocytes. Eur J Pharmacol 532: 201-207, 2006.

41. Lim JH, Wen TC, Matsuda S, Tanaka J, Maeda N, Peng H, Aburaya J, Ishihara K and Sakanaka M: Protection of ischemic hippocampal neurons by ginsenoside Rb1, a main ingredient of ginseng root. Neurosci Res 28: 191-200, 1997. 
42. Tian J, Fu F, Geng M, Jiang Y, Yang J, Jiang W, Wang C and Liu K: Neuroprotective effect of 20(S)-ginsenoside Rg3 on cerebral ischemia in rats. Neurosci Lett 374: 92-97, 2005.

43. Zhao H, Li Q, Zhang Z, Pei X, Wang J and Li Y: Long-term ginsenoside consumption prevents memory loss in aged SAMP8 mice by decreasing oxidative stress and up-regulating the plasticity-related proteins in hippocampus. Brain Res 1256: 111-122, 2009.

44. Liu ZQ, Luo XY, Liu GZ, Chen YP, Wang ZC and Sun YX: In vitro study of the relationship between the structure of ginsenoside and its antioxidative or prooxidative activity in free radical induced hemolysis of human erythrocytes. J Agric Food Chem 51: 2555-2558, 2003.

45. Liu ZQ, Luo XY, Sun YX, Chen YP and Wang ZC: Can ginsenosides protect human erythrocytes against free-radical-induced hemolysis? Biochim Biophys Acta 1572: 58-66, 2002.

46. Liu D, Zhang H, Gu W, Liu Y and Zhang M: Neuroprotective effects of ginsenoside Rb1 on high glucose-induced neurotoxicity in primary cultured rat hippocampal neurons. PLoS One 8: e79399, 2013.

47. Liu Z, Chen J, Huang W, Zeng Z, Yang Y and Zhu B: Ginsenoside $\mathrm{Rb} 1$ protects rat retinal ganglion cells against hypoxia and oxidative stress. Mol Med Rep 8: 1397-1403, 2013.

48. Tan SJ, Yu Z and Dong QT: Effects of ginsenoside Rb1 on the oxidative stress in the skeletal muscles of rats with postoperative fatigue syndrome. Zhongguo Zhong Xi Yi Jie He Za Zhi 32: 1535-1538, 2012 (In Chinese).

49. Wu Y, Xia ZY, Dou J, Zhang L, Xu JJ, Zhao B, Lei S and Liu HM: Protective effect of ginsenoside Rb1 against myocardial ischemia/reperfusion injury in streptozotocin-induced diabetic rats. Mol Biol Rep 38: 4327-4335, 2011.
50. Migliore L and Coppedè F: Environmental-induced oxidative stress in neurodegenerative disorders and aging. Mutat Res 674: 73-84, 2009.

51. Li J, O W, Li W, Jiang ZG and Ghanbari HA: Oxidative stress and neurodegenerative disorders. Int J Mol Sci 14: 24438-24475, 2013.

52. Xie Y, Zhao QY, Li HY, Zhou X, Liu Y and Zhang H: Curcumin ameliorates cognitive deficits heavy ion irradiation-induced learning and memory deficits through enhancing of Nrf2 antioxidant signaling pathways. Pharmacol Biochem Behav 126 181-186, 2014.

53. Li W and Kong AN: Molecular mechanisms of Nrf2-mediated antioxidant response. Mol Carcinog 48: 91-104, 2009.

54. Li B, Choi HJ, Lee DS, Oh H, Kim YC, Moon JY, Park WH, Park SD and Kim JE: Amomum tsao-ko Suppresses lipopolysaccharide-induced inflammatory responses in RAW264.7 macrophages via Nrf2-dependent heme oxygenase-1 expression. Am J Chin Med 42: 1229-1244, 2014.

55. Was H, Dulak J and Jozkowicz A: Heme oxygenase-1 in tumor biology and therapy. Curr Drug Targets 11: 1551-1570, 2010.

56. Wu ML, Ho YC, Lin CY and Yet SF: Heme oxygenase-1 in inflammation and cardiovascular disease. Am J Cardiovasc Dis 1: 150-158, 2011.

57. Tkachev VO, Menshchikova EB and Zenkov NK: Mechanism of the Nrf2/Keap1/ARE signaling system. Biochemistry (Mosc) 76: 407-422, 2011.

58. Zenkov NK, Menshchikova EB and Tkachev VO: Keap1/Nrf2/ARE redox-sensitive signaling system as a pharmacological target. Biochemistry (Mosc) 78: 19-36, 2013.

59. Dinkova-Kostova AT, Holtzclaw WD and Kensler TW: The role of Keap1 in cellular protective responses. Chem Res Toxicol 18: 1779-1791, 2005 